\title{
Labyrinthe
}

4 | 1999

Numéro 4

\section{La Vierge au voile}

Étude iconographique

\section{Anne Malherbe}

\section{CpenEdition}

Journals

Édition électronique

URL : http://journals.openedition.org/labyrinthe/292

DOI : $10.4000 /$ labyrinthe.292

ISSN : 1950-6031

Éditeur

Hermann

\section{Édition imprimée}

Date de publication : 1 octobre 1999

Pagination : 59-72

\section{Référence électronique}

Anne Malherbe, « La Vierge au voile », Labyrinthe [En ligne], 4 | 1999, mis en ligne le 19 février 2005,

consulté le 30 avril 2019. URL : http://journals.openedition.org/labyrinthe/292 ; DOI : 10.4000/

labyrinthe.292

Ce document a été généré automatiquement le 30 avril 2019.

Propriété intellectuelle 


\title{
La Vierge au voile
}

\author{
Étude iconographique
}

\author{
Anne Malherbe
}

1 En 1419, avec l'assassinat de Jean sans Peur, duc de Bourgogne, la rupture s'accomplit entre le royaume de France et le duché de Bourgogne. Ce duché possède les Pays-Bas depuis le mariage de Philippe le Hardi et de Marguerite de Flandre, en 1369 : c'est là que la cour s'installe alors. Sous le coup de ces événements, l'activité artistique se déplace de Paris (qui passe sous la coupe des Anglais dès 1419) vers les Pays-Bas, et se caracqxtérise par une production d'œuvres qui émanent directement de la Cour et de son entourage. La nouvelle sorte de commanditaires que sont les foncqxtionnaires de cour favorise le développement de techniques picturales neuves (celle, en particulier, de la peinture à l'huile et du glacis) et la mise au point d'iconographies liées à la dévotion. Des tableaux propres à la dévotion privée constituent la majeure partie de la demande - forme de dévotion dont le Duc et des courants religieux comme la « Dévotion Moderne » montrent l'exemple.

2 Ces œuvres sont destinées à prendre place dans la partie de la maiqxson que le particulier réserve à la dévotion, et elles sont consacrées aux oraisons individuelles. En général, pour les œuvres qui nous sont parveqxnues, il s'agit de peintures à l'huile sur panneau de bois, de petit format.

3 L'iconographie se rapporte à ce qui, dans le culte, fait l'objet d'une dévoqxtion particulièrement prisée (souvent directement rattachée aux indulqxgences papales). Philippe le Bon, duc de Bourgogne depuis 1419, montre une dévotion toute particulière à la Vierge et en répand le culte dans le duché. Sous cette influence, les tableaux qui présentent la Vierge se mulqxtiplient. Ces ouvrages ont une forme qui leur est propre. De fait, ils doiqxvent placer le dévot en relation directe avec le Divin, et avec le personnage montré (Christ, Vierge, saint). Leur fonctionnement n'est pas très éloigné de celui de l'icône, puisque les représentations sont analogues à des signes qui renverraient à une réalité supérieure. Mais en outre, les peintres n'hésitent pas à exploiter les possibilités toutes nouvelles qu'offre la peinture à l'huile pour accroître l'illusionnisme de la représentation. Il faut que le dévot puisse prendre modèle sur l'image qu'il contemple (le 
principe de l'imitation est fondamental dans la Dévotion moderne) et qu'il ressente intensément la présence du Christ ou du saint. L'émotion, l'empathie, voire le contact sensible avec l'objet de la dévotion, sont eux aussi essenqxtiels à la dévotion. Il en résulte des cadrages resserrés et des compositions dans lesquelles les personnages semblent vouloir communiquer avec le dévot.

Le début du XVI ${ }^{\mathrm{e}}$ siècle verra naître des changements dans la peinqxture. D'une part, parce que le centre artistique se déplace de Bruges-Bruxelles à Anvers (lors du mariage de Marguerite de Bourgogne avec Maximilien, archiduc d'Autriche), mais aussi en raison de la peinture floqxrentine (Léonard de Vinci en particulier), qui, par l'intermédiaire des voyages d'artistes, est mieux connue et suscite des préoccupations nouqxvelles. Il n'empêche que, en dépit d'un modelé différent des visages (la Vierge que nous allons étudier en est un exemple), la composition et le rôle dévotionnel des œuvres perdurent souvent. Les tableaux continuent (du moins jusque vers 1530) à offrir des motifs à la contemplation des dévots.

\section{VIERGE AU VOILE}

5 À une date incertaine, mais probablement aux alentours de 1550, est peinte une série de panneaux qu'on intitule la Vierge à l'Enfant entortillé ou la Vierge au Voile. Cette série est sans doute issue de l'atelier ou de l'entourage de Paul Coeck d'Alost (fils de Pierre Coeck d'Alost), du milieu anversois ${ }^{1}$. L'un de ces panneaux est conservé à Anvers, au Musée Royal des Beaux-Arts². La Vierge, richement vêtue de velours rouge et vert, avec des garnitures d'or, est visible à mi-corps derrière un parapet. Sa position est en léger trois quarts. Une tenture, derrière son dos, semble indiquer qu'elle est assise, trônant sous un dais. En tout cas, le décor paraît être la loggia d'un palais, donc un espace à la fois luxueux, ouvert, délimité et préservé. Une colonnette (de marbre vraisemblablement) est placée entre le parapet de pierre, dans le fond du tableau, et l'architrave supérieure (invisible). Sur ce même parapet est posé un vase de lis, tandis qu'une poignée de cerises est répandue sur le parapet du premier plan. C'est sur ce rebord que l'Enfant est assis et que s'accoude la Vierge. Tous deux regarqxdent le spectateur. L'Enfant Jésus sourit.

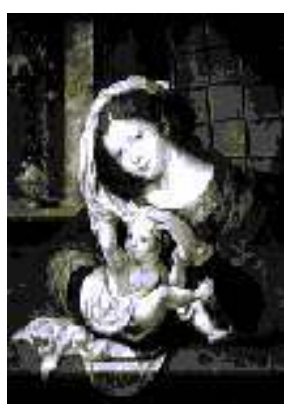

III. : Vierge à l'Enfant au voile entortillé attribuée au Maître des demi-figures huile sur bois, $68 \times 56$

Anvers, Musée des Beaux-Arts INV. 183

6 Notre attention a tout particulièrement été retenue par le voile de la Vierge qui s'enroule autour de l'Enfant, devenant ainsi à la fois voile et linge. Le mouvement du voile crée un axe vertical dans la composition. De la tête de la Vierge, le voile descend sur celle de l'Enfant. Celui-ci le souqxlève de ses deux mains. Puis il passe derrière le dos de Jésus, enserre sa taille et, glissant au-dessus de ses jambes, il décrit un dernier coude pour s'étaler enfin entre l'Enfant et le parapet. 
7 Ce tableau n'est pas le seul de la série qui nous soit parvenu. Nous en avons recensé quatre autres qui présentent une iconographie similaire ${ }^{3}$. Le décor, cependant, varie selon les panneaux. Au lieu de la loggia, c'est une pièce ouverte sur l'extérieur par une fenêtre - la fenêtre laisse voir, mais non à chaque fois, le paysage du dehors. Dans deux cas, les lis sont placés avec les cerises sur le parapet du premier plan. Dans un autre, les deux motifs ont disparu. Dans le dernier cas, une pomme remplace le vase avec les fleurs. Seuls demeurent toujours identiques la position de la Vierge à l'Enfant et le mouvement du voile, qui a éveillé notre curiosité*.

8 Ce voile est-il un ornement ou une variation, à fins esthétiques, sur le voile de la Vierge ? Dit-il quelque chose de la Vierge et de l'Enfant? Invite-t-il à une dévotion particulière?

Rendons compte avant tout des éléments connus.

10 Le décor où la Vierge se tient semble interchangeable. C'est cepenqxdant à chaque fois un intérieur. Ce genre de décor n'est pas nouveau aux Pays-Bas. Dès les années 1460-1470 ${ }^{4}$ à Bruges, à Bruxelles, il se substiqxtue souvent au fond neutre devant lequel, chez Van der Weyden en partiqxculier, se tient la Vierge. Cette formule correspond à la coutume des dipqxtyques avec dévot (à l'origine de laquelle est Van der Weyden), où celui-ci prie au milieu d'un cadre qui lui est familier.

11 Est-ce à dire que ce décor recherche le réalisme ou la vraisemblance ? Dans deux cas, la fenêtre ouvre sur un paysage désertique. Un âne et deux personnages, dans le fond, suggèrent la représentation d'une fuite en Égypte.

12 Le motif du parapet n'est pas nouveau lui non plus. Déjà présent à la fin $d u X^{\mathrm{e}}$ siècle, il se répand dans le milieu anversois au début du $X I^{e}$ siècle, notamment dans les représentations de la sainte Famille avec cadrage rapproché ou dans les Vierges du Rosaire $^{5}$. Il tend à créer un lieu privilégié où la Vierge, proche mais à l'écart, reçoit l'hommage du dévot. C'est un espace idéal, familier par son aspect, mais qui est transcendé en réalité par un ordre autre que terrestre. Le parapet est aussi le lieu où la Vierge offre l'Enfant à la dévotion. Elle l'assoit dessus afin de le présenter au dévot. Dans notre exemple, l'ostension du corps de l'Enfant est marquée également par la musculature développée de l'Enfant et par son sourire.

Près du couple divin, d'autres éléments connus occupent l'espace : les cerises, qui disent la Passion que le Christ subira, les pommes, indicaqxtion du rachat, par cette même Passion, de la faute originelle, les lis, qui attestent la virginité de Marie ou son Immaculée Conception $^{6}$.

14 Au sein de cet ensemble, le voile se rattache-t-il à l'un ou l'autre des sens déjà présents ? Ajoute-t-il une signification nouvelle?

Examinons si des antécédents formels du motif ne sauraient fournir quelques indices.

16 L'étoffe qui crée un lien visible entre la Vierge et l'Enfant existait auparaqxvant. En nous restreignant aux Pays-Bas bourguignons, citons le panneau central du Retable Bladelin, de Rogier van der Weyden (Berlin-Dahlem, Gemäldegalerie), des années 1450, qui représente une Nativité. Le manqxteau bleu de la Vierge (ici ce n'est donc pas son voile) sert de drap sur lequel l'Enfant est couché. De même, dans l'Annonciation de Jan van Eyck, conservée à la National Gallery de Washington et datant des années 1420-1430, la Vierge a posé son livre (préfiguration du Verbe incarné qu'est l'Enfant) sur un pan de son manteau. Ainsi la Vierge crée-t-elle pour Jésus un lit de son vêtement. Elle manifeste qu'elle a porté le Christ (ou va le porter) dans son sein. Le thème suggéré par le motif est 
ici celui de la maternité et donc de l'Incarnation : l'Enfant fut porté dans le ventre de la Vierge.

Toujours par Van der Weyden, dans les années 1450, les Vierge à l'Enfant à mi-corps voient unis par leur blancheur éclatante, se faisant l'un à l'autre écho, le voile de la Vierge (qui passe par-dessus son sein découqxvert) et le linge dans lequel Marie porte l'Enfant ${ }^{7}$. Comme dans le panneau d'Anvers qui nous occupe, l'Enfant sourit (en regardant vers le donateur situé sur le panneau adjacent). Notons que la Vierge et l'Enfant sont entouqxrés de rayons dans les panneaux de Caen et de Tournai.

Des antécédents plus directs sont perceptibles chez Jan Gossaert. Selon Friedländer ${ }^{8}$, la Vierge au Voile entortillé aurait pour original une œuvre de Gossaert, des années 1510-1530. Friedländer fonde son asserqxtion sur l'étude formelle du visage de Marie. Nulle preuve supplémentaire ne permet de confirmer le propos. Il est vrai que l'on connaît de Gossaert des exemples de Vierge dont le voile s'enroule autour de l'Enfant mais de façon moins complexe que dans les panneaux qui nous intéressent. Au Mauristhuis de La Haye se trouve une composition où l'Enfant est assis sur un coin de table derrière laquelle se tient Marie. Le voile de Marie retombe sur la tête de Jésus, qui le retient de ses mains, et descend jusque sur la table. Friedländer ${ }^{9}$ date le tableau d'avant 1520. Un autre panneau (Munich, marché de l'art, cité par Friedländer ${ }^{10}$ ) représente une Vierge à l'Enfant devant un cadre en trompe-l'œil. Le voile de la Vierge passe au-dessus de sa poitrine, se prolongeant jusqu'à l'aisselle droite. Le linge sur lequel Marie tient l'Enfant, sans être un pan du voile, en crée l'illusion. L'Enfant tient une grenade dans sa main. À la même époque, d'autres ouvrages révèlent une insistance particulière sur le motif du voile, avec des variantes entre une œuvre et une autre. Barend van Orley par exemple fait lui aussi du linge de l'Enfant le prolongement illusoire du voile de Marie, dans une représentation de la Vierge et l'Enfant s'embrassant qui daterait des alentours de $1518{ }^{11}$. L'insistance sur le voile semble ne pas s'accompagner toujours du même thème, ce qui en complique l'interprétation. Le voile, dont on constate l'inflation et qui semble s'imposer plus qu'auparavant au regard du dévot, serait-il alors l'objet de variations à seules fins esthétiques? Un simple motif décoratif, se développant sous l'effet d'un phénomène de mode ? De nouvelles pratiques de productions, à ce même moment, à Anvers, facilite ce genre de phénomènes. Comme l'explique

G. Marlier ${ }^{12}$, les peintres du début du XVI ${ }^{e}$ siècle ont l'idée d'organiser leurs ateliers de telle sorte que les panneaux pourraient être produits en série à l'instar des gravures. La forte demande d'œuvres pour l'exportation (vers l'Espagne, les pays scandinaves, l'Allemagne, etc.) incite elle aussi à cette pratique et à la composition d'ouvrages où les motifs, loin de resqxsortir à une dévotion ou à une commande particulières, se développent en fonction de leur succès commercial. Le voile de la Vierge en serait-il le résultat ? Ou existe-t-il une cause précise qui en aurait suscité l'existence et l'aurait chargé d'un sens?

Nous nous sommes demandé si, au début du $\mathrm{XVI}^{\mathrm{e}}$ siècle, n'aurait pas été ravivé un élément particulier de la dévotion envers Marie. Un sondage effectué dans les textes liés à la dévotion aux Pays-Bas - la Légende dorée, de Jacques de Voragine ${ }^{13}$, les ouvrages proches de la Dévotion Moderne $e^{14}$, ceux des mystiques rhénans ${ }^{15}$, dans les récits de miracles ${ }^{16}-$ ne nous ont rien apporté. Nous nous sommes tourné alors vers d'autres pistes.

21 Notre attention a été retenue par certaines représentations en bois sculpté qui se répandent dans le Trentin au début du XVI ${ }^{e}$ siècle, sous l'effet d'influences germaniques ${ }^{17}$. 
Il s'agit en particulier de deux Vierges, ornant la partie centrale de retables de bois. L'une a été réalisée vers 1500, par l'atelier de Narciso da Bolzano (l'ouvrage est conservé à Trente, Museo proqxvinciale d'Arte) ; l'autre vers 1520, dans l'entourage de Jörg Lederer (Trente, Museo diocesano tridentino). Ces deux Vierges sont des exemples de Maria in sole, debout sur la lune et entourée de rayons. Toutes deux portent l'Enfant et leur voile connaît là encore une extension remarquable, passant sous l'Enfant (dans l'un des deux cas il longe également la poiqxtrine de Marie) et s'échappant en vrilles vers le bas. Ces retables particuqxliers - ou d'autres retables présents dans la même région - n'ont pas nécessairement eu une influence directe sur les tableaux qui nous occuqxpent. Toutefois, nous pouvons les rattacher à plusieurs faits.

Aux alentours des années 1500, se développe à Anvers le marché des retables à caisse de bois sculptée et à volets peints (autrement dit, comme ceux que nous venons de voir), qui sont le fruit de plusieurs ateliers. Cette production donnait lieu à des foires mais aussi à des congrès où se renconqxtraient des artistes d'origine très diverses ${ }^{18}$ et où les peintres pouvaient côtoyer des artistes étrangers et voir leurs ouvrages. L'iconographie de ces Vierges a pu se répandre par ce moyen mais grâce, aussi, à la gravure.

À la même époque, dans les Pays-Bas, se diffuse dans la peinture sur panqxneau l'iconographie de Maria in sole, sous l'impulsion que provoquent des indulgences émises par Sixte IV. En 1476, Sixte IV introduit l'office de l'Immaculée Conception. Dans des bulles de 1476 et 1477, il promet des indulgences nouvelles à ceux qui célébreraient cet office. Or la repréqxsentation de Maria in sole est liée à ce culte ${ }^{19}$.

Devons-nous lui rattacher aussi le motif du voile entortillé ? L'iconographie des retables de bois y invite. Regardons de plus près le motif. Il unit étroitement la Vierge et l'Enfant et les encercle tous deux, de la même manière, dans les circonvolutions blanches du voile. Alors que les panneaux cités plus hauts, où la Vierge, derrière un parapet, présente l'Enfant au dévot, placent la Vierge en position secondaire par rapport à l'Enfant, le motif $\mathrm{du}$ voile semble accorder une place similaire aux deux personnages. Le regard de la Vierge est dirigé, comme celui de Jésus, vers le dévot, alors que traditionnellement Marie tient ses yeux baissés.

Quand le manteau ou le voile protège l'Enfant, nous l'avons dit, il atteste que Marie a porté l'Enfant dans son sein. Le voile est blanc : Marie est Vierge. Ce voile, accordant ici un même statut à la Vierge et à Jésus, dit sans doute que Marie est née elle aussi sans souillure, d'une mère vierge. Le voile, unissant les deux personnages, exprime une qualité sainte qui leur est commune : leur engendrement d'une mère vierge.

Un panneau de Memling, conservé à la Alte Pinakothek de Munich et peint vers 1480, abonde en ce sens. Il présente sainte Anne, la Vierge et l'Enfant, dans un espace étroit qui pourrait passer pour une loggia (de chaque côté, deux colonnettes semblent border une ouverture sur l'extérieur). Anne trône. À ses pieds est agenouillée Marie, qui tient ellemême l'Enfant sur ses genoux. Le mouvement du manteau d'Anne est remarqxquable, qui enveloppe Marie et sert de linge où asseoir l'Enfant. La posiqxtion d'Anne, frontale, dans laquelle s'imbriquent les deux autres personqxnages, laisse à penser qu'on a affaire à une représentation de l'Immaculée Conception. Encore une fois, le motif de l'étoffe s'enroulant autour d'un personnage apparaît significatif.

Par ailleurs, l'association, dans les Vierge à l'Enfant (dont la postérité a été vaste) de Van der Weyden, du motif du voile et du linge blancs avec les rayons qui auréolent la tête des 
deux personnages a peut-être contribué à rattacher le motif du voile à l'iconographie de la Vierge in sole ${ }^{20}$.

La forme que décrit le voile doit-elle être lue comme un indice ? Le voile dessine un double S (deux S dont la base de l'un forme le sommet de l'autre), autrement dit, par deux fois, la première lettre du mot Sole - par un phénomène de redondance qui accentue l'effet visuel du motif. La préqxsence du lis confirme peut-être ce sens. Le Cantique des Cantiques $(2,2)$, cité par Molanus, associe les images de la lune, du soleil et de lis, pour déqxcrire la Bien-aimée. Ainsi le lis ressortit à la représentation de l'Immaculée Conception et non pas seulement à celle de la virginité de Marie.

Le motif du voile, sa forme en $\mathrm{S}$, le vase de lis s'associeraient alors pour créer le sens de cette composition, pour en faire une représentation nouvelle, particulière, de l'Immaculée Conception ${ }^{21}$. Cependant, parmi les compositions recensées, il en est deux d'où les fleurs sont absentes. Le voile se vide-t-il, dans ces deux cas, de tout contenu ? Le motif est-il alors assez connu pour n'avoir plus besoin d'être éclairci par un autre motif ? Le sens de l'œuvre en est-il modifié ? Se nuance-t-il d'une autre signification?

Nous avons vu que les compositions avec la Vierge au Voile comportent des cerises, signe de la Passion, parfois aussi une pomme, qui indique le rachat, par la Passion, de la faute originelle. Dans l'un des ouvrages de Gossaert que nous avons cités à titre de comparaison, l'insistance sur le voile de Marie est accompagnée de la représentation d'une grenade (fruit, là encore, de la Passion). Le sourire de l'Enfant, son aspect physique semqxblent marquer l'Incarnation. Le voile se connote-t-il d'un sens proche de ceux-ci ?

31 La blancheur du voile, le fait qu'il enlace l'Enfant et, surtout, sa longueur inusitée le désignent comme un linceul, celui-là même qui a recouvert le Christ. De même que les fruits - ainsi, du reste, que la représentation de la Fuite en Égypte, qui, liée au massacre des innocents, annonce le marqxtyre du Christ - préfigurent la Passion, le long voile rend déjà présent le sacrifice du Christ. La superposition du voile et du linceul n'est pas nouqxvelle aux Pays-Bas. Et il se peut que les œuvres citées plus haut, qui émaqxnent du milieu de Jan Gossaert, apparentent déjà les deux motifs. En effet, dans les années 1515 apparaît dans la peinture sur panneau, chez Quentin Metsys en particulier - donc toujours dans le milieu anversois - le thème de l'Enfant assoupi, préfiguration connue, là encore, de la mort du Christ. Sur un panneau du Musée des Beaux-Arts de Tournai, la Vierge tient sur ses genoux l'Enfant qui dort. Sur ses cheveux, elle porte un genre de turban blanc, prolongé par un pan de la même étoffe. Celui-ci glisse le long du sein découvert de la Vierge et enveloppe l'Enfant.

La mise en valeur du voile, sa place proprement centrale dans la composition surprennent dans le panneau d'Anvers, et dans ceux de la même série. Or, c'est dans la première moitié du XVI ${ }^{\mathrm{e}}$ siècle que le saint Suaire de Turin commence de connaître un réel renom ${ }^{22}$.

En 1506, Jules II prononce des indulgences plénières liées à la dévotion envers la relique. Plus tard, dans la nuit du 3 au 4 décembre 1532, un incendie fait rage dans la Sainte Chapelle de Chambéry où le Suaire est conservé. La relique est sauvée et, en dépit de marques laissées par les flammes, l'image du corps est intacte. L'événement, considéré comme miraculeux, provoque la fortune définitive du Suaire, désormais tenu pour le linceul véritable du Golgotha. Clément VII, à l'issue d'une enquête qu'il fait mener, confirme par une nouvelle bulle les indulgences déjà accordées par Jules II. 
Se pourrait-il que l'insistance sur le voile et le lien qu'il entretient avec l'iconographie de la Passion, faisant de lui une préfiguration du Suaire, soit à rattacher au succès de la relique et aux indulgences papales? Ce fait n'est pas à exclure. Il faudrait alors revoir la datation des œuvres. Les premières compositions où le voile et le linge portant l'Enfant font l'objet d'un traitement particulier (dans le milieu de Gossaert, de Metsys) pourraient se situer après que Jules II eut accordé des indulgences. Et la série de compositions qui nous occupe suivrait alors l'épisode de l'incendie, de la confirmation des indulgences, en somme, de l'entière reconnaissance du Suaire comme celui du Christ.

L'examen précis du motif révèle que le voile, depuis la tête de la Vierge et en passant par l'Enfant, s'avance progressivement vers le specqxtateur pour finalement retomber devant le parapet, s'offrant ainsi pleineqxment à l'adoration. De tous les motifs de la composition, c'est celui qui est le plus proche du dévot. Il est de tous le premier à recevoir l'hommage.

3 Le traitement illusionniste du motif qui semble pouvoir être touché par le dévot, atteint par son baiser d'adoration, est le substitut même de la relique. Présent comme il l'est dans l'œuvre, il n'est pas seulement l'image du Suaire : la dévotion dont il fait l'objet doit posséder une efficacité comparable à celle de la relique véritable. Et c'est pourquoi sans doute se mulqxtiplient les œuvres qui le représentent, permettant à tous d'obtenir sa part d'indulgence.

Cette autre signification possible du voile exclut-elle la précédente? Si, dans toutes ces compositions, l'Enfant n'est point endormi mais regarde le dévot avec vivacité, si le mouvement $\mathrm{du}$ voile décrit réellement un double $\mathrm{S}^{23}$ et si les fruits de la Passion s'associent avec les lis, c'est que le voile se charge conjointement des deux sens. Le motif, véritable invention picqxturale, est issu de plusieurs courants. Les représentations traditionnelles de la Vierge dans le Soleil, celles de l'Enfant endormi ou du linge compris comme un linceul, celles qui, par souci esthétique, visent à varier la coifqxfure de la Marie. Des faits extérieurs, telles les indulgences, ont été l'occasion de mettre au point le motif. Des nuances peuvent modifier celui-ci, selon que les lis sont ou non représentés, selon peut-être aussi les intenqxtions de prière du dévot. À ce voile n'est pas rattaché un sens définitif et strictement délimité, mais sa forte présence picturale lui permet d'attirer le regard, de le voir d'abord comme un avènement visuel que le dévot considérera comme doté d'une réalité divine et transcendante. C'est alors que l'entourage - celui de la composition même puis celui de la dévotion - suggère des significations, crée des modulations. Mais le voile, surtout, conduit le regard de l'ordre terrestre à l'ordre divin, vers Marie et Jésus ; puis d'une figure à l'autre, de la Vierge à l'Enfant, et inversement. Car au fond c'est cela qui importe le plus : obtenir, par la contemplation, la grâce que le regard de la Vierge, celui de l'Enfant ainsi que son sourire, incarnent déjà.

BIBLIOGRAPHIE

FRIEDLÄNDER M. J., Early Netherlandish Painting. From Van Eyck to Bruegel, Londres, 1965. 
MARLIER G., La Renaissance flamande. Pierre Coeck d'Alost, Bruxelles, 1966.

PANOFSKY E., Early Netherlandish Painting. Its Origin and Character, Cambridge, Mass., 1953, 2 vol. ; rééd., 1 vol., Harvard, 1971 ; trad. fr., Paris, 1992.

- Peinture et dévotion à la fin du Moyen Âge, Paris, 1997 pour la traducqxtion française.

PÉRIER D'IETEREN C., « Le marché d'exportation et l'organisation du traqxvail dans les ateliers brabançons au XV et $\mathrm{XVI}^{\mathrm{e}}$ siècles. Apport de l'examen technologique des retables ", dans Artistes, Artisans et Production artistique au Moyen Âge, Colloque international, CNRS, Université de Rennes II-Haute-Bretagne 2 - 6 mai 1983, vol. III, p. 629-646.

PнiLIPРот P., La Peinture dans les Anciens Pays-Bas, XV -XVIe siècles, Paris, 1994.

RINGBOM S., "Maria in Sole and the Virgin of the Rosary ", Journal of the Warbourg and Courtauld Institutes, XXV, 1962, p. 326-330.

- Icon to narrative. The Rise of the dramatic close-up in fifteenth-century devotional painting, $2^{\mathrm{e}}$ édition revue et augmentée, The Netherlands, [s.1.], 1983.

- Les Images de dévotion (XII ${ }^{e}-\mathrm{XV}^{e}$ siècle), Paris, 1995 pour la traduction française. [Rassemblement de trois textes publiés antéqxrieurement : Devotional Images and Imaginative Devotions Notes on the place of Art in late medieval private Piety ", Gazette des Beaux-Arts, mars 1969 ; « Some pictorial Conventions for the Recounting of Thoughts and Experiences in late medieval Art ", Medieval Iconography and Narrative, a Symposium, Odense, 1980 ; « Vision and Conversation in early Netherlandish Painting : the Delft Master's Holy Family », Simiolus, 1989.]

WiLSON J. C., « Marketing Paintings in late medieval Flanders and Brabant », dans Artistes, Artisans et Production artistique au Moyen Âge, Colloque international, CNRS, Université de Rennes II-HauteBretagne 2-6mai 1983, vol. III, p. 621-628.

\section{NOTES}

1.Ces informations, ainsi que les œuvres connues de cette série sont tirées de G.Marlier, La Renaissance flamande, Pierre Coeck d'Alost, Bruxelles, 1966, p. 245-47. Cet ouvrage est le seul à réaliser une synthèse des ouvrages du milieu de Pierre Coeck et de sa postérité, c'est-à-dire des années 1530-1550 à Anvers. Les œuvres sont étudiées par types de composition, après une mise au point sur le contexte politique et écoqxnomique de la production. L'étude est principalement formelle, visant à la constitution d'un catalogue. 2.Le panneau est, dans ce cas, attribué par le musée d'Anvers au Maître des Demi-figures, première moiqxtié du XVIe siècle. Cette œuvre n'est pas signalée par Marlier. Cependant elle est fort semblable à celles qu'il présente. C'est aussi la seule que nous ayons pu voir de près. Nous signalons également que nous en avons vu un autre exemple - attribué à J. Gossaert, mais que ne recensent ni G. Marlier (op. cit.) ni M. J. Friedländer (Early Netherlandish Painting, Leyde-Bruxelles, 1972) - à la Pinacoteca nazionale de Lucca, en Toscane.

3.Ces panneaux sont présentés par G.Marlier, op. cit. et par M. J. Friedländer, op. cit.

*. Cet article a été effectué dans le cadre d'un travail de maîtrise portant sur la peinture

flamande aux XV $\mathrm{XV}^{\mathrm{e}}$ XVI $\mathrm{X}^{\mathrm{e}}$ siècles, sous la direction de Daniel Russo à Paris IV-Sorbonne, en 1997. 
4.Voir notamment une Vierge à l'Enfant de Dierick Bouts, à la National Gallery de Londres, datant des environs de 1465. On y retrouve le parapet et le décor d'intérieur. C'est aussi le cas dans la Vierge avec Martin van Nieuwenhove d'Hans Memling, à l'hôpital Saint-Jean de Bruges, datant de 1487.

5.L'œuvre de Joos Van Cleve fournit plusieurs exemples : La Vision de saint Bernard, à Paris, musée du Louvre (la Vierge et le saint sont derrière le parapet ; l'Enfant est assis dessus et tient un rosaire) ; la Sainte Famille, à Rome, Collection Odescalchi, construite sur un même modèle. Nous nous contentons de citer ceux-ci.

6.Si la présence traditionnelle des lis dans les Annonciations tend à faire d'eux les symboles de la virgiqxnité de Marie, notons que Molanus, dans son Traité des Saintes Images (rédigé en 1568-1569, édité pour la dernière fois en traduction française à Paris, en 1996) rattache le lis à l'Immaculée Conception ( $c f$. III, 55, 375, où l'image du lis est associée à celle de la lune et du soleil, propres à la représentation de l'Immaculée Conception). Reste à savoir si Molanus, exposant ce qui lui semble convenable, enregistre ou non un état de fait.

7.Cf. les Vierge à l'Enfant de l'Institute of Fine Arts de Chicago, du Musée des Beaux-Arts de Caen, du Musée des Beaux-Arts de Tournai.

8.Cité par G.Marlier, op. cit., p. 245.

9.Max J.Friedländer, op. cit., pl. 31.

10.Ibid.

11.Cité par Max J.Friedländer, op. cit., pl. 117.

12.G.Marlier, op. cit., p. 15.

13.Jacques de Voragine, La Légende dorée, 2 tomes, traduit par J.-B. M. Roze, Paris, 1967.

14.Thomas à Kempis, Vita Jesu Christ, éd. par Parisiis et typographia regia, 1640 ; Bonaventure (saint) [pseudo-Bonaventure], Méditations de la Vie du Christ, traduit par Henri de Riancey, Paris, 1900 ; Ludolphe le Chartreux, La Grande Vie de Jésus-Christ, traduit par le père Dom Florent Broquin, religieux du même ordre, Paris, 1891 ; Bernard de Clairvaux (saint), « À la louange de la Vierge Mère », dans Sources chrétiennes, traduction M. I. Huille et J. Regnard, Paris, 1993.

15.Rusbrock dit L'Admirable, CEuvres choisies, traduites par E. Hello, textes conformes à l'édition de 1869, Plan de la Tour (Var), 1984 ; Brigitte de Suède (sainte), Révélations, traduit du latin et introduit par B. Baudonnel, Nouan-le-Fuzelier, 1991 ; Henri de Suso, Euvres complètes, présentation, traduction et notes de J. Ancelet-Hustache, Paris, 1977. 16. Miracles et mystères : la littérature religieuse au nord de la France, textes présentés et traduits par F. J. Beaussart, M. de Combarieu du Grès, J. Subrenat, Paris, 1989.

17. Ces ouvrages sont cités dans Imago linea. Scultura lignea nel Trentino dal XII al XVI secolo, a cura di E. Castelnuovo, Milan, 1986. Par l'examen d'un catalogue d'œuvres, sont mis au jour les courants d'échanges artistiques entre certains pays germaniques et la région du Trentin.

18.Voir à ce sujet l'intervention de C. Périer d'Ieteren, « Le marché d'exportation et

l'organisation du travail dans les ateliers brabançons aux $\mathrm{XV}^{\mathrm{e}}$ et XVI $\mathrm{X}^{\mathrm{e}}$ siècles. Apports de l'examen technologique des retables » dans Artistes, Artisans et Production artistique au Moyen Âge, colloque international, CNRS, Université de Rennes II-Haute-Bretagne, 2-6 mai 1983, vol. III, p. 629-645. C. Périer d'Ieteren suit de façon très étroite les résultats fournis par l'étude des retables de manière à réaliser une étude poussée des techniques de production de ces ouvrages.

19.Cf. S.Ringbom, " Maria in Sole and the Virgin of the Rosary ", Journal of the Warbourg and Courtauld Institutes, XXV, 1962, p. 326-330. À partir d'un triptyque de Gérard David, 
conservé à la National Gallery de Londres, Ringbom étudie comment, vers 1500, se combinent dans une même œuvre l'iconographie du Rosaire et celle de l'Immaculée Conception, sous l'effet d'indulgences émises par Sixte IV. C'est de cet article que nous tirons nos renseignements sur la dévotion envers l'Immaculée Conception.

20.Les Vierge à l'Enfant de Van der Weyden semblent trop précoces pour être des représentations de la Vierge in sole. Les peintres postérieurs ont pu cependant retenir l'idée d'associer les rayons de lumière à l'insistance sur la blancheur du voile et du linge, et tirer de là une interprétation particulière des deux motifs.

21.Nous nous sommes demandé si le Concile de Trente avait insisté sur la question de l'Immaculée Conception, contribuant ainsi à l'invention et à la fortune de l'iconographie que nous étudions. Mais il ne semble pas que la question ait été fortement discutée (cf. Dictionnaire de Spiritualité, ascétique et mysqxtique, doctrine et histoire, article « Marie », vol. X (1), col. 457, Paris, 1977).

22.Nous tenons les informations sur le Suaire de l'étude de F. Lecercle, « De la relique à

l'image : la promotion du Suaire de Turin ", dans Symboles de la Renaissance, III $^{\mathrm{e}}$ vol., Paris, 1990, p. 95-112. L'auteur s'attache principalement à la promotion du Suaire dans la

littérature au début du XVII ${ }^{e}$ siècle et s'efforce d'approfondir la notion de relique pour ce qui est d'une relique aussi exceptionnelle que le Suaire.

23.La lettre $\mathrm{S}$ est bien la première lettre du mot sole, mais aussi celle de Sindon, le mot latin désignant le suaire

\section{RÉSUMÉS}

Au milieu du XVI ${ }^{\mathrm{e}}$ siècle, une série particulière de tableaux de dévoqxtion apparaît dans le milieu anversois. Tous présentent la même iconographie, celle de la Vierge dont le voile s'enroule avec complexité autour de l'Enfant. Ce voile, récurrent d'une œuvre à l'autre, semble s'offrir à la contemplation et à l'adoration. Une enquête menée dans le contexte dévotionnel - dans les pratiques et dans les sources textuelles - ainsi que la confrontation à d'autres représentations mettent en lumière le sens possible de ce voile. Celui-ci désignerait la Vierge comme Vierge de l'Immaculée Conception, et, en même temps, il serait le substitut illuqxsionniste du saint Suaire, alors particulièrement vénéré.

The Virgo with the Veil. Iconographic study. - In the middle of the sixteenth century, a particular series of devotion picqxtures appear in the Antwerp artistic milieu. All present the same iconograqxphy, i.e. the one of the Virgo, whose veil winds with complexity around the Child. This veil, recurrent from one work to another, seems to offer itself for contemplation and adoration. An inquiry conducted in the devotional context - in the practices and in the textual sources - as well as the confrontation with representation lights up the possible meaning of the veil. The latter may designate the Virgo as the Virgo of the Immaculate Conception, and meanwhile it may be the illusionist substitute to the holy shroud of the Christ, which was particularly venerated at that time. 


\section{AUTEUR}

\section{ANNE MALHERBE}

Anne MALHERBE, élève de l'ENS-Ulm, agrégée de Lettres modernes, a réalisé une maîtrise, en histoire de l'art, portant sur l'iconographie du souqxrire dans la peinture sur panneau des anciens Pays-Bas flamands (1420-1530). Actuellement, elle achève un DEA d'histoire de l'art contemporain, sur la matérialité dans l'œuvre de Paul Rebeyrolle. 